\title{
A NOTE ON INHOMOGENEOUS DIOPHANTINE APPROXIMATION WITH A GENERAL ERROR FUNCTION
}

\author{
AI-HUA FAN \\ Department of Mathematics, Wuhan University, Wuhan, Hubei, 430072, P.R. China and \\ LAMFA, CNRS UMR 6140, Université de Picardie, 80039 Amiens, France \\ e-mail: aihua.fan@u-picardie.fr \\ and JUN WU \\ Department of Mathematics, Huazhong University of Science and Technology, \\ Wuhan, Hubei, 430074, P.R. China and \\ LAMFA, CNRS UMR 6140, Université de Picardie, 80039 Amiens, France \\ e-mail:wujunyu@public.wh.hb.cn
}

(Received 22 November, 2004; revised 17 January, 2006; accepted 10 February, 2006)

\begin{abstract}
Let $\alpha$ be an irrational number and $\varphi: \mathbb{N} \rightarrow \mathbb{R}^{+}$be a decreasing sequence tending to zero. Consider the set

$$
E_{\varphi}(\alpha)=\{\beta \in \mathbb{R}:\|n \alpha-\beta\|<\varphi(n) \text { holds for infinitely many } n \in \mathbb{N}\},
$$

where $\|\cdot\|$ denotes the distance to the nearest integer. We show that for general error function $\varphi$, the Hausdorff dimension of $E_{\varphi}(\alpha)$ depends not only on $\varphi$, but also heavily on $\alpha$. However, recall that the Hausdorff dimension of $E_{\varphi}(\alpha)$ is independent of $\alpha$ when $\varphi(n)=n^{-\gamma}$ with $\gamma>1$.
\end{abstract}

2000 Mathematics Subject Classification. 11J83, 28A80.

1. Introduction. Let $\alpha$ be an irrational real number. Minkowski [9] showed that the inequality

$$
\|n \alpha-\beta\|<\frac{1}{4 n}
$$

has infinitely many solutions for any $\beta$ which is not in the orbit of $\alpha$, i.e. $\beta \notin \mathbb{Z}+\alpha \mathbb{Z}$. In general the constant $\frac{1}{4}$ is the best possible (see [4]). In 1999, Bernik and Dodson [1, p. 105] considered the set of real numbers which are well approximated by $(\{n \alpha\})_{n \geq 1}$ :

$$
E_{\gamma}(\alpha)=\left\{\beta \in \mathbb{R}:\|n \alpha-\beta\|<\frac{1}{n^{\gamma}} \text { holds for infinitely many } n \in \mathbb{N}\right\}
$$

where $\gamma>1$ and $\{\cdot\}$ denotes the fractional part. They proved that the Hausdorff dimension of $E_{\gamma}(\alpha)$ satisfies

$$
\frac{1}{\omega \gamma} \leq \operatorname{dim} E_{\gamma}(\alpha) \leq \frac{1}{\gamma}
$$


where $\omega \geq 1$ is any positive number for which

$$
\|n \alpha\| \geq \frac{1}{n^{\omega}} \quad \text { for all sufficiently large integers } n .
$$

This, together with Khintchine's theorem, see [3, Theorem 1.10], implies that for almost all real numbers $\alpha$ (with respect to the Lebesgue measure)

$$
\operatorname{dim} E_{\gamma}(\alpha)=\frac{1}{\gamma}
$$

In 2003, Bugeaud [2], Schmeling and Troubetzsky [10] improved, independently, the above result due to Bernik and Dodson as follows.

THEOREM 1 (Bugeaud-Schmeling-Troubetzsky). For any irrational number $\alpha$,

$$
\operatorname{dim} E_{\gamma}(\alpha)=\frac{1}{\gamma}
$$

Schmeling and Troubetzsky [10] used Theorem 1 and an inhomogeneous version of Jarník-Besicovitch theorem to show some strong recurrence properties of the billiard flow in certain polygons.

In this note we would like to know what happens when $n^{-\gamma}$ is replaced by a general decreasing sequence. Let $\varphi: \mathbb{N} \rightarrow \mathbb{R}^{+}$be a function satisfying the following hypothesis

$$
\varphi(n) \downarrow 0 \text { as } n \rightarrow \infty, \quad n \varphi(n) \leq \frac{1}{2} \text { for large } n .
$$

Let $\alpha$ be an irrational number. Consider the set

$$
E_{\varphi}(\alpha)=\{\beta \in \mathbb{R}:\|n \alpha-\beta\|<\varphi(n) \text { holds for infinitely many } n \in \mathbb{N}\} .
$$

It may be proved that for almost all real numbers $\alpha$,

$$
\operatorname{dim} E_{\varphi}(\alpha)=\limsup _{n \rightarrow \infty} \frac{\log n}{-\log \varphi(n)} .
$$

In fact, the lower bound of $\operatorname{dim} E_{\varphi}(\alpha)$ can be deduced from [1, Theorem 5.1] and the arguments in [1, p. 105]. The upper bound is a consequence of Theorem 1 (see also the formula (6) below). The formula (4) also holds when $\alpha$ is an irrational number with bounded partial quotients (see the remark at the end of the note).

All these results show that the formula (4) doesn't depend on $\alpha$ in all cases studied. However, as we shall prove, in general the Hausdorff dimension of $E_{\varphi}(\alpha)$ depends not only on $\varphi$, but also heavily on $\alpha$. The formula (4) is not always true.

THEOREM 2. There exist an irrational number $\alpha_{0}$ and a function $\varphi: \mathbb{N} \rightarrow \mathbb{R}^{+}$ satisfying $(\mathrm{H})$ such that

$$
\limsup _{n \rightarrow \infty} \frac{\log n}{-\log \varphi(n)}>0, \text { but } \operatorname{dim} E_{\varphi}\left(\alpha_{0}\right)=\liminf _{n \rightarrow \infty} \frac{\log n}{-\log \varphi(n)}=0 .
$$


2. Proof of Theorem 2. Define

$$
l(\varphi)=\limsup _{n \rightarrow \infty} \frac{\log n}{-\log \varphi(n)}, \quad u(\varphi)=\liminf _{n \rightarrow \infty} \frac{\log n}{-\log \varphi(n)} .
$$

These quantities $l(\varphi)$ and $u(\varphi)$ are closely related to the upper and lower orders at infinity of $1 / \varphi$, used by Dodson [6] (see also Dodson [7] and Dickinson [5]) to generalize the Jarník-Besicovitch theorem. We remark that it is easy to deduce from Theorem 1 that for any irrational number $\alpha$,

$$
u(\varphi) \leq \operatorname{dim} E_{\varphi}(\alpha) \leq l(\varphi)
$$

For a given irrational number $\alpha \in(0,1)$, let $\left[0 ; a_{1}, a_{2}, \cdots, a_{n}, \cdots\right]$ be the simple continued fraction expansion of $\alpha$. The convergents are obtained via finite truncations

$$
\frac{p_{n}}{q_{n}}:=\left[0 ; a_{1}, a_{2}, \cdots, a_{n}\right]
$$

With the convention $p_{-1}=q_{0}=1, q_{-1}=p_{0}=0$, we have the well known recursive relations

$$
\begin{array}{cl}
p_{n}=a_{n} p_{n-1}+p_{n-2} & \text { for } n \geq 1, \\
q_{n}=a_{n} q_{n-1}+q_{n-2} & \text { for } n \geq 1 .
\end{array}
$$

We are now going to construct our desired number $\alpha_{0} \in(0,1)$ and sequence $(\varphi(n))_{n \geq 1}$ satisfying $(\mathrm{H})$.

Construct $\alpha_{0}=\left[0 ; a_{1}, a_{2}, \ldots, a_{n}, \ldots\right]$ by choosing $a_{n}$ in the following recursive way,

$$
a_{1}=1, \quad a_{n+1}=2^{3 q_{n}} \quad \text { for any } n \geq 1
$$

where the $q_{n}$ are recursively determined by (8). For any $k \geq 1$, write

$$
N_{k}=q_{k-1}+q_{k}+a_{k+1}^{1 / 3} \cdot q_{k}-1 .
$$

Let $1<\gamma<2$. Define

$$
\varphi(n)=\frac{1}{N_{k}^{\gamma}}, \text { if } N_{k-1}<n \leq N_{k} .
$$

It is easy to check that

$$
l(\varphi)=\frac{1}{\gamma}, \quad u(\varphi)=0 .
$$

It remains to prove $\operatorname{dim} E_{\varphi}\left(\alpha_{0}\right)=0$ for the above defined $\alpha_{0}$ and $\varphi$. At first we prove that $\operatorname{dim}\left(E_{\varphi}\left(\alpha_{0}\right) \bigcap[0,1)\right)=0$. Obviously we can $\operatorname{regard} \mathbb{R} / \mathbb{Z}$ as $[0,1)$. For any $k \geq 1$, we consider the finite sequence of points $\left\{n \alpha_{0}\right\}$ with $q_{k-1}+q_{k} \leq n<q_{k}+q_{k+1}$. The distribution of these points is well described by Three Distance Theorem (see Halton [8] or Slater [11]). Since $q_{k}+q_{k+1}=q_{k-1}+q_{k}+a_{k+1} \cdot q_{k}$ (see (8)), any $n$ satisfying $q_{k-1}+q_{k} \leq n<q_{k}+q_{k+1}$ can be written as

$$
n=q_{k-1}+q_{k}+t \cdot q_{k}+m \text {, with } \quad 0 \leq t<a_{k+1} \quad \text { and } \quad 0 \leq m<q_{k} .
$$


For any fixed $0 \leq m<q_{k}$, we call $\left\{\left(q_{k-1}+q_{k}+t \cdot q_{k}+m\right) \alpha_{0}, 0 \leq t<a_{k+1}\right\}$ the $m$ th subsequence. Thus the finite sequence $\left\{n \alpha_{0}, q_{k-1}+q_{k} \leq n<q_{k}+q_{k+1}\right\}$ can be decomposed into $q_{k}$ subsequences of length $a_{k+1}$ corresponding to $m=0,1, \ldots, q_{k}-1$. For any fixed $0 \leq m<q_{k}$, consider the set of points

$$
A_{k}(m):=\left\{\left\{\left(q_{k-1}+q_{k}+i \cdot q_{k}+m\right) \alpha_{0}\right\}, i=0,1, \cdots, a_{k+1}^{1 / 3}-1\right\}
$$

which consists of the first $a_{k+1}^{1 / 3}$ points in the $m$ th subsequence and $\bigcup_{m=0}^{q_{k}-1} A_{k}(m)$ coincides with $\left\{n \alpha_{0}\right\}$ for which $q_{k-1}+q_{k} \leq n \leq N_{k}$. Observe that the distance of two consecutive points in $A_{k}(m)$ satisfies

$$
\begin{aligned}
& \left\|\left(q_{k-1}+q_{k}+(i+1) \cdot q_{k}+m\right) \alpha_{0}-\left(q_{k-1}+q_{k}+i \cdot q_{k}+m\right) \alpha_{0}\right\| \\
& \quad=\left\|q_{k} \alpha_{0}\right\|<\frac{1}{q_{k+1}}<\frac{1}{a_{k+1} \cdot q_{k}} .
\end{aligned}
$$

It follows that for any two points $x, y \in A_{k}(m)$ we have

$$
\|x-y\|<\frac{1}{a_{k+1}^{2 / 3} \cdot q_{k}} .
$$

When $k$ is large enough, we have $\frac{1}{a_{k+1}^{2 / 3} \cdot q_{k}} \leq N_{k}^{-\gamma}$. Thus for large $k$ we have

$$
\bigcup_{i=0}^{a_{k+1}^{1 / 3}-1} B\left(\left\{\left(q_{k-1}+q_{k}+i \cdot q_{k}+m\right) \alpha_{0}\right\}, N_{k}^{-\gamma}\right) \subset B\left(\left\{\left(q_{k-1}+q_{k}+m\right) \alpha_{0}\right\}, 3 N_{k}^{-\gamma}\right),
$$

where $B(x, r)$ denotes the ball with centre $x$ and radius $r$. Consequently, for any integer $n \geq 1$ the set $E_{\varphi}\left(\alpha_{0}\right) \bigcap[0,1)$ is contained in

$$
\bigcup_{k=n}^{\infty}\left(\bigcup_{m=0}^{q_{k}-1} B\left(\left\{\left(q_{k-1}+q_{k}+m\right) \alpha_{0}\right\}, 3 N_{k}^{-\gamma}\right) \bigcup \bigcup_{p=N_{k}+1}^{q_{k}+q_{k+1}-1} B\left(\left\{p \alpha_{0}\right\}, N_{k+1}^{-\gamma}\right)\right) \text {. }
$$

Then, for any $s>0$, by the definitions of $\alpha_{0}$ and $\varphi$, we can estimate the $s$ dimensional Hausdorff measure as follows:

$$
\begin{aligned}
\mathcal{H}^{s}\left(E_{\varphi}\left(\alpha_{0}\right) \cap[0,1)\right) & \leq \liminf _{n \rightarrow \infty} \sum_{k=n}^{\infty}\left[q_{k}\left(6 N_{k}^{-\gamma}\right)^{s}+\left(a_{k+1}-a_{k+1}^{1 / 3}\right) \cdot q_{k} \cdot\left(2 N_{k+1}^{-\gamma}\right)^{s}\right] \\
& \leq \liminf _{n \rightarrow \infty} \sum_{k=n}^{\infty}\left[q_{k}\left(6 N_{k}^{-\gamma}\right)^{s}+a_{k+1} \cdot q_{k} \cdot\left(2 N_{k+1}^{-\gamma}\right)^{s}\right]=0 .
\end{aligned}
$$

Thus $\operatorname{dim}\left(E_{\varphi}\left(\alpha_{0}\right) \cap[0,1)\right)=0$. Since $E_{\varphi}\left(\alpha_{0}\right)$ is invariant under translations by $\mathbb{Z}$, so the full theorem follows from the conclusion in the unit interval.

Using the similar idea as that in Bugeaud [2], Schmeling and Troubetzsky [10], we can get the following result (the details are omitted).

THEOREM 3. If $\alpha$ is an irrational number with bounded partial quotients and $\varphi$ : $\mathbb{N} \rightarrow \mathbb{R}^{+}$is a function satisfying $(H)$, we have

$$
\operatorname{dim} E_{\varphi}(\alpha)=u(\varphi)
$$




\section{REFERENCES}

1. V. I. Bernik and M. M. Dodson, Metric diophantine approximation on manifolds, Cambridge Tracts in Mathematics 137 (Cambridge University Press 1999).

2. Y. Bugeaud, A note on inhomogeneous Diophantine approximation, Glasgow Math. J. 45 (2003), 105-110.

3. Y. Bugeaud, Approximation by algebraic numbers, Cambridge Tracts in Mathematics 160 (Cambridge University Press 2004).

4. J. W. S. Cassels, An introduction to Diophantine approximation, Cambridge Tracts in Math. and Math. Phys. 99 (Cambridge University Press 1957).

5. H. Dickinson, A remark on a theorem of Jarník, Glasgow Math. J. 39 (1997), 233-236.

6. M. M. Dodson, Hausdorff dimension, lower order and Khintchine's theorem in metric Diophantine approximation, J. Reine Angew. Math. 432 (1992), 69-76.

7. M. M. Dodson, Geometric and probabilistic ideas in the metrical theory of Diophantine approximation, Russian Math. Surveys 48 (1993), 73-102.

8. J. H. Halton, The distribution of the sequence $\{n \xi\}(n=0,1,2, \ldots)$, Proc. Cambridge Phil. Soc. 61 (1965), 665-670.

9. H. Minkowski, Diophantische Approximationen (Teubner: Leipzig, Berlin 1907).

10. J. Schmeling and S. Troubetzkoy, Inhomogeneous Diophantine approximation and angular recurrence properties of the billiard flow in certain polygons, Math. Sbornik 194 (2003), 295-309.

11. N. B. Slater, Gaps and steps for the sequence $n \theta \bmod 1$, Proc. Cambridge Phil. Soc. 63 (1967), 1115-1123. 